\section{A GAME TO HELP STUDENTS COMPREHEND ENZYME STRUCTURE AND MECHANISM OF ACTION}

\section{DONALD E. HULTQUIST}

Department of Biological Chemistry, Medical School The University of Michigan

Ann Arbor, Michigan 48104
Conference-recitation periods may successfully serve to review and integrate lecture material, but they often fail to excite and motivate the student. In searching for alternative techniques with which a biochemistry lecture course can be supplemented, I have found that active participation of students in a game is an efficient and enjoyable way for them to comprehend enzymology. The game is presented here to encourage others to use this technique.

The students were enrolled at the University of Michigan in a one-semester introductory biochemistry course either for undergraduates or for students in the Integrated Premedical-Medical Program. Prior to playing the game, students had listened to lectures and read chapters in textbooks on proteins and enzymology, including the structure and mechanism of action of proteases. Two groups of ten students played the game at one time. Each group was placed in a small room with a set of space-filling molecular models, instructions on how to use the models, a previously built backbone of an $\alpha$-helix, and biochemistry textbooks.

The instructor explained that the ten students were being asked to accomplish a task as a group, and that the other group was working on the same problem. It was stressed that in order to complete the exercise in one hour, they would have to rapidly organize themselves and make efficient use of their collective knowledge, deductive abilities, and creativity. Each student was directed to read the "Information and Instruction Sheet" and then to proceed according to its directions. The instructur left the room until the work was well underway and during the remainder of the hour helped only in the techniques of model building. The instructor insured that the groups had selected one or two speakers for the oral presentations which were subsequently made to the combined groups. A visiting "expert" along with the instructor, moderated the session, corrected errors, reminded the students to distinguish between deduction and hypothesis, and kept criticism and arguments under control.

Each time this game was played during the past five years, the majority of the students became intensely involved in the exercise and learned a great deal from one another. The excitement apparently stems from the group dynamics and the strong competition which quickly arises between the two groups. Results of questionnaires returned anonymously by $85 \%$ of the students during the past two years showed that $66 \%$ considered that "the enzyme game was fun and an effective learning device", while $23 \%$ disagreed with this statement. From having worked with these ideas and models, the students may very well retain the knowledge longer and have a better sense of the reality of enzymes. I recommend this technique as an effective way to reinforce lecture material. The usefulness of the technique appears to be limited only by the quality of the game presented to the class. The variations for such biochemical games are clearly unlimited.

\section{Information and Instruction Sheet for the Enzyme Game}

Suppose that during the past summer the students in this section had received a research grant to study the biochemical basis of a dystrophic disease that affected the muscles in early childhood. The results of an intensive summer of research by your group resulted in the finding that extremely high levels of a previously unrecognized cathepsin (proteolytic enzyme) were present in the muscles from patients with this disease.

Moreover, the group found that this enzyme showed an unusual specificity toward small peptides - it hydrolyzed only those peptide bonds in which both the carboxyl and amino groups were provided by L-aspartic acid residues. Much to everyone's surprise, a peptide containing 22 amino acid residues, which was obtained by tryptic digestion of the cathepsin, showed $34 \%$ of the specific activity of the native enzyme and showed the same strict substrate specificity. Other studies suggested that the specificity resulted from two binding sites for anions at the active site of the enzyme. ORD studies of the 22 amino acid residue peptide established that $91 \%$ of the peptide was in a helical form.

Amino acid analysis gave the following composition for the peptide:

tyr-1 ala-2 leu-1 met-1 ser-1 glu-3 his-2 phe-1 gly-2 ile-1 val-4 asp-2 lys-1

Treatment of the 22-residue peptide with cyanogen bromide yielded two peptides - a pentapeptide containing one residue each of Leu, Gly, His, Met, and Ser. The larger peptide contained a $\mathrm{N}$-terminal histidine. Neither peptide showed catalytic activity.

Initial studies on the amino acid sequence of the 22 residue peptide showed that the two histidyl residues were separated by at least two residues. The $\mathrm{pH}$ dependency of the activity is shown for both the native enzyme and the peptide in the following figure.

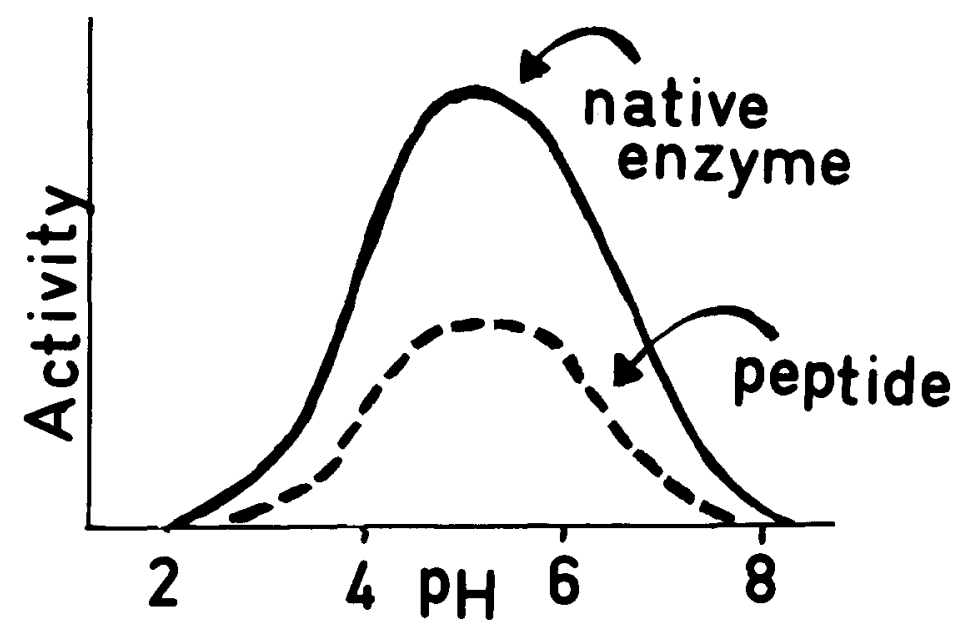

Preliminary studies of the mechanism of action suggested that an acyl enzyme was an intermediate in the enzyme-catalyzed reaction.

Assume that later this afternoon a representative of the granting agency will make a visit to determine whether money should be made available for the continuation of this project. Working as two independent teams for the next 60 minutes: (1) build a model of a substrate for the enzyme, (2) build a model of a possible active site of the enzyme, and (3) propose a reasonable mechanism of action for the enzyme. Each group will have 10 minutes to make a presentation.

It will probably be necessary for each group to break up into subgroups in order to complete the different tasks (namely, to build the substrate, to build the side chains of the active site, and to postulate a structure for the active site of the enzyme that takes into consideration all of the available data). Since atoms are limiting, choose wisely what structure should be built. Information on the active sites of chymotrypsin and carboxypeptidase may be of value to you. You may wish to refer to texts. Don't let failure of theoreticians and workers to communicate be your downfall. A practice presentation where the group can criticize the postulated active site and mechanism would be valuable before the final presentation is made. 